\title{
Changes in Gait and Radiographic and Clinical Results of Calcaneal Lengthening Osteotomy in Children with Idiopathic Flexible Flatfoot
}

\author{
Ha Yong Kim, MD, Yong Han Cha, MD, Jong Shin Lee, MD, \\ Kyung-Min Kil, MD*, Won Sik Choy, MD \\ Department of Orthopaedic Surgery, Eulji University Hospital, Daejeon, \\ ${ }^{*}$ Department of Orthopaedic Surgery, National Police Hospital, Seoul, Korea
}

Background: In this study, we aimed to analyze kinematic and kinetic changes in gait and to assess radiographic and clinical improvement after calcaneal lengthening osteotomy (CLO) in children with idiopathic symptomatic flexible flatfoot deformities.

Methods: The study group consisted of 22 children (28 feet) with idiopathic symptomatic flexible flatfoot, which was treated by CLO as an index operation. We excluded patients with flatfoot that developed secondary to neuromuscular diseases. There were 18 boys (22 feet) and 4 girls ( 6 feet). The average age of the patients was $10.8 \pm 1.51$ years. Surgical results were assessed by radiologic imaging, clinical evaluation, and gait analysis, which were performed before surgery and 1 year after surgery. Medical records were reviewed to check postoperative complications.

Results: Talonavicular coverage was recovered to $16.7^{\circ}$ from $32.7^{\circ}(p<0.01)$. Lateral talo-first metatarsal angle and calcaneal pitch angle increased by $20.1^{\circ}$ and $9.9^{\circ}$, respectively $(p<0.01)$. Weight-bearing radiographs demonstrated a decrease in the anteroposterior angle and lateral talocalcaneal angle by $6.9^{\circ}$ and $10^{\circ}$, respectively $(p<0.01)$. Hindfoot valgus was corrected to near neutral position after CLO. Kinematic results showed that the ankle valgus angle in the coronal plane was reduced from $35.48^{\circ}$ preoperatively to $16.64^{\circ}$ postoperatively during gait $(p<0.05)$. The preoperative out-toeing gait (preoperative foot progression angle, $20.31^{\circ}$ ) was also normalized to $14.21^{\circ}$ postoperatively. The kinetics results showed that the push-off moment (maximum ankle plantar flexion) on the sagittal plane increased from $0.66 \mathrm{Nm} / \mathrm{kg}$ preoperatively to $0.83 \mathrm{Nm} / \mathrm{kg}$ postoperatively $(p<0.01)$. The mean Yoo score was $9.2 \pm 1.05$ postoperatively: satisfactory in 24 cases $(86 \%)$ and unsatisfactory in 4 cases. Of the 4 unsatisfactory cases, 2 cases were overcorrection and 2 cases were undercorrection, and 3 of them were well managed by wedge foot insoles.

Conclusions: The 3-dimensional deformities of the flexible flatfoot were effectively corrected by CLO, based on the morphological assessment using radiologic imaging. The correction was well maintained during gait at 1 year after the operation.

Keywords: Idiopathic flexible flatfoot, Calcaneal lengthening osteotomy, Gait analysis, Kinematics, Kinetics

Flatfoot is a commonly observed 3-dimensional foot deformity combined with hindfoot valgus, reduced medial longitudinal arch height, and forefoot abduction. ${ }^{1,2)}$ Mid-

Received October 25, 2019; Accepted January 13, 2020

Correspondence to: Yong Han Cha, MD

Department of Orthopaedic Surgery, Eulji University Hospital, 95 Dunsanseo-

ro, Seo-gu, Daejeon 35233, Korea

Tel: +82-42-611-3280, Fax: +82-42-611-3283

E-mail: naababo@hanmail.net foot breakage causes a decrease in the hardness of the lever. Forefoot abduction and heel valgus cause the propulsive force to be less effective as the muscles act in a different direction to the lever at a certain angle. In terms of kinematics, the flatfoot leads to lever arm dysfunction due to the flexible, short lever arm. ${ }^{3,4)}$ Thus, to produce push-off moments on the sagittal plane during gait, the gastrocnemius-soleus complex should generate more force to compensate for the ineffective lever arm in the flatfoot. ${ }^{5,6)}$ This 
Kim et al. Effect of Calcaneal Lengthening in Idiopathic Flexible Flatfoot

Clinics in Orthopedic Surgery • Vol. 12, No. 3, $2020 \bullet$ www.ecios.org

increased load on the triceps muscle leads to muscle fatigue in long distance walking, resulting in various clinical symptoms. Kim et al. ${ }^{7)}$ reported that patients with severe pes planovalgus walked with decreased moment (by approximately $30 \%$ ) and decreased power (by approximately $45 \%$ ) compared to individuals with normal feet.

For the treatment of symptomatic flexible flatfoot in children, conservative treatments are tried first such as rest, medication, physical therapy, and use of an insole. However, if the symptoms persist after conservative treatment, surgical treatment can then be considered. The most common surgical methods are soft-tissue procedures, osteotomies, subtalar arthrodesis and arthroeresis. ${ }^{8}$ Among these, calcaneal lengthening osteotomy (CLO) is a procedure that can simultaneously correct the heel valgus and forefoot abduction and increase the calcaneal pitch and longitudinal arch by lengthening the lateral column of the foot, which tightens the peroneus longus tendon and plantar fascia and blocks abduction of navicular at the talonavicular joint. Thus, CLO is the most physiologic surgical procedure for normal anatomical alignment.

There have been many reports that investigated the effects of conservative and surgical treatments on flatfoot in clinical and radiological aspects. ${ }^{5,69)}$ However, there are only a few quantitative studies on mechanical disadvantages or postoperative kinematic and kinetic changes in flexible flatfoot, and their subjects were limited to cerebral palsy patients. ${ }^{2,410-12)}$ Thus, we aimed to analyze the kinematic and kinetic changes of gait and to assess the clinical and radiologic improvements in children treated by CLO for moderate to severe symptomatic flexible flatfoot deformities that had not been improved with conservative treatment for several months.

\section{METHODS}

The study design and protocol were approved by the Institutional Review Board of the Eulji University Hospital (IRB No. EMC 2019-02-012). Informed consent was waived because of the retrospective nature of this study.

\section{Subjects}

The subjects of this retrospective study were children with symptomatic flexible flatfoot who underwent CLO at our institution from October 2011 to February 2015. The inclusion criteria were as follows: (1) CLO was performed as the primary operation and patients were followed up for more than 1 year after surgery. If the patient had both feet problems, CLO was followed up for more than 1 year after last surgery. (2) Patients were evaluated by radiologic imaging, and gait analysis was performed before and after surgery. (3) CLO was performed for children before the skeletal maturity (age, 8 to 13 years). Subjects with flatfoot that had developed due to neuromuscular diseases (cerebral palsy, myelomeningocele, and poliomyelitis), tarsal coalition, and congenital vertical talus were excluded. In addition, we excluded patients who had undergone lower leg operations, such as derotational osteotomies of the femur or tibia, which affect the kinetics or kinematics of gait analysis. However, patients who underwent lengthening of gastrosoleus muscle or Kidner's operation that was required for correction of flatfoot deformity were included in the study (Fig. 1).

A total of 22 patients (28 feet) were included in the study: 18 were boys ( 22 feet) and 5 were girls ( 6 feet). There were 5 patients with both feet problems, 15 patients with right foot problems, and 3 patients with left foot problems. Their mean age was $10.8 \pm 1.51$ years. The patients had been treated conservatively for more than 6 months before surgery, with no improvement. Major complaints of these patients were limping gait (18 patients) and pain (11 patients). The location of the pain was the heel, medial arch, and accessory navicular area.

\section{CLO}

CLO was performed with a combination of lateral softtissue release, calcaneal osteotomy, bone graft, and internal fixation, depending on the patient's condition. Surgery was performed by a senior surgeon (HYK). In patients with both feet problems, the interval between operations was 6 months to 1 year. A transverse skin incision of $3 \mathrm{~cm}$ was made along the superior border of the calcaneus from the anterior calcaneal process to the peroneal tendons posteriorly. The peroneus brevis tendon was lengthened with Z-plasty while protecting the sural nerve. Then, CLO was performed between the anterior facet and the middle facet of the subtalar joint. The osteotomy site was distracted with a lamina spreader and bone grafts were placed in the distracted gap. For the bone grafts, we used allobone chips, tri-

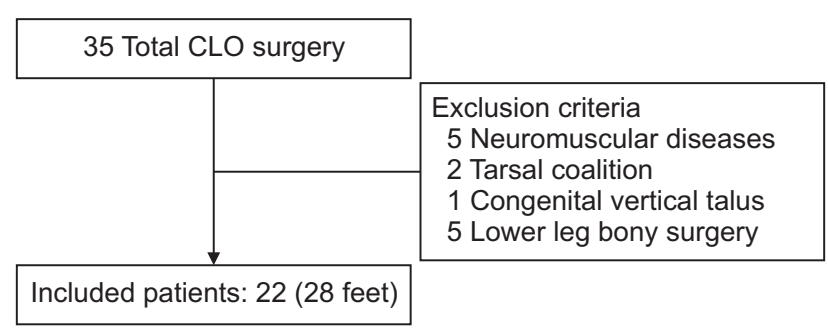

Fig. 1. Flowchart of study inclusion. CLO: calcaneal lengthening osteotomy. 
Kim et al. Effect of Calcaneal Lengthening in Idiopathic Flexible Flatfoot

Clinics in Orthopedic Surgery • Vol. 12, No. 3, $2020 \bullet$ www.ecios.org

cortical allobone, or autobone from the accessory navicular. The osteotomy site was fixated by the plate (7 feet) or staple (14 feet) according to the stability of the fragment. When tricortical allograft was used, internal fixation was not performed. Achilles contracture, accessory navicular, and hallux valgus were corrected simultaneously when CLO was performed: tendo-Achilles lengthening, 7 cases; vulpius procedure, 14 cases; Strayer procedure, 1 case; Kidner's operation, 11 cases; and correction of hallux valgus, 2 cases.

\section{Radiographic Evaluation}

To assess the degree of static deformity of the flatfoot, anteroposterior and lateral radiographs of the foot were taken in standing position. Radiographs taken preoperatively and 1 year after surgery were used for measurement. The following parameters were measured on radiographs: (1) anteroposterior talonavicular angle (AP-TNA) and anteroposterior talo-first metatarsal angle (AP-T1MTA) for evaluation of forefoot abduction on anteroposterior radiographs; (2) lateral T1MTA (L-T1MTA, Meary angle) and lateral calcaneal pitch angle (L-CPA) for evaluation of the medial longitudinal arch on lateral radiographs; and (3) anteroposterior talocalcaneal angle (AP-TCA, Kite's angle) and lateral TCA (L-TCA) for evaluation of hindfoot valgus (Fig. 2). Radiographic measurements were performed by 2 orthopedic surgeons (YHC and KMK) using the software of Picture Archiving Communication System (PACS; Marosis m-view, ver. 5.4, Infinitt, Seoul, Korea). To reduce measurement errors, measurements were taken twice by each surgeon, and the average values were calculated. We were able to achieve intraobserver and interobserver reliability of 0.94 and 0.95 , respectively.

\section{Gait Analysis}

The gait analysis was performed preoperatively and 1 year after surgery. If the patient had both feet problems, postoperative gait analysis was performed at 1 year after last surgery. Ankle range of motion and kinematic and kinetic data were obtained by analysis. Gait analysis was done using the Eagle camera system (Eagle system, Motion Analysis, Santa Rosa, CA, USA) and the AMTI force plate (AMTI, Watertown, MA, USA). Passive reflective markers were attached using the modified Helen-Hayes method. EvaRT ver. 4.2 (Motion Analysis) and Orthotrak (Motion Analysis) software were used for data processing to obtain kinematic and kinetic data. While the children were walking at a comfortable speed on the walkway several times, optical tracking and motion capture were done 120 times per second $(120 \mathrm{~Hz})$. The ground reaction force was measured using the AMTI force plate, and the moments and

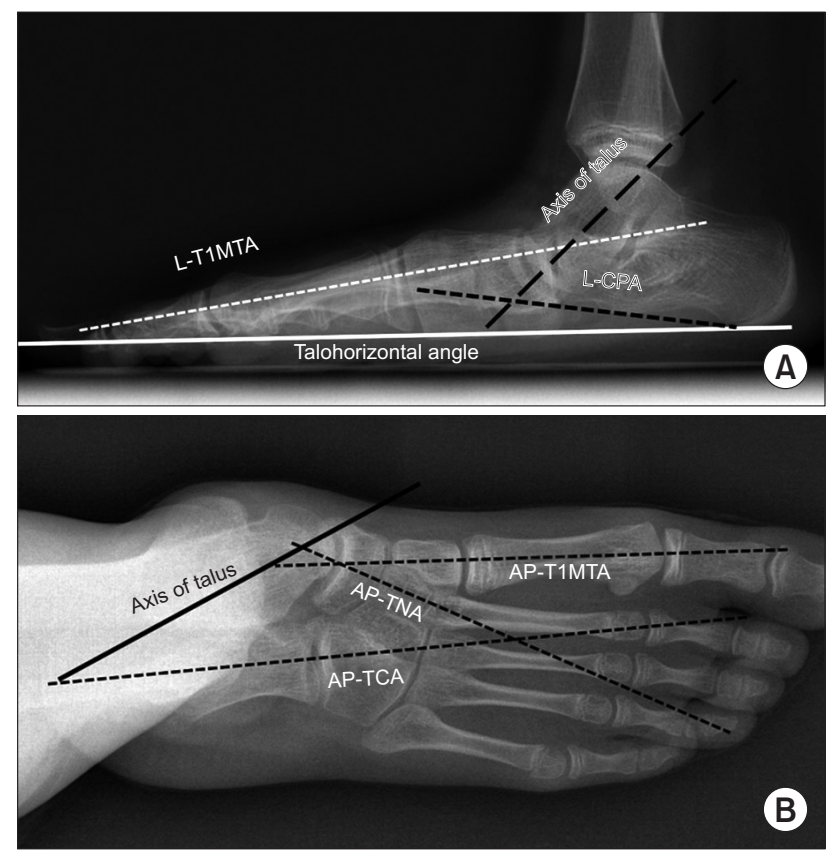

Fig. 2. Radiographic measurements. (A) Standing foot lateral radiograph. (B) Standing anteroposterior radiograph. L-T1MTA: lateral talo-first metatarsal angle (Meary angle), L-CPA: lateral calcaneal pitch angle, AP-T1MTA: anteroposterior talo-first metatarsal angle, AP-TNA: anteroposterior talonavicular angle, AP-TCA: anteroposterior talocalcaneal angle.

forces applied to each joint were calculated by software. The gait analysis was repeated 5 times and after excluding the minimum and maximum values, the means of the remaining 3 values were used. The mean value, maximum, minimum, and ranges on the kinetic and the kinematic graph during gait were used for the statistical analysis.

\section{Clinical Evaluation}

Ankle range of motion was measured using a goniometer, and the degrees of ankle plantar flexion and dorsiflexion were recorded. Ankle dorsiflexion was measured with the knee in extension (ankle dorsiflexion with knee in extension [ADKE]) and flexion (ankle dorsiflexion with knee in flexion $[\mathrm{ADKF}]$ ).

The scoring system suggested by Yoo et al. ${ }^{10)}$ was used to assess the following 4 parameters after surgery: pain, forefoot abduction, medial arch, and hindfoot valgus. Three points were given for each of the 4 items: $\geq 8$ points was classified as satisfactory, and $<7$ points was classified as unsatisfactory. Postoperative complications were also investigated by reviewing medical records.

We regarded undercorrection as Meary angle less than $5^{\circ}$ without symptomatic improvement. For overcorrection, the postoperative foot shape was cavovarus foot with Meary angle more than $10^{\circ}$ and symptomatic callosi- 
Kim et al. Effect of Calcaneal Lengthening in Idiopathic Flexible Flatfoot

Clinics in Orthopedic Surgery • Vol. 12, No. 3, $2020 \bullet$ www.ecios.org

ties under the lateral column of the foot.

\section{Statistical Analysis}

A paired $t$-test was used to compare the preoperative and postoperative radiographic measurements and the kinetics and kinematics data. The level of significance was set at $p<$ 0.05 (SPSS ver. 14.0; SPSS Inc., Chicago, IL, USA).

\section{RESULTS}

\section{Radiographic Evaluation}

The mean AP-TNA improved from $32.7^{\circ} \pm 11.8^{\circ}$ to $16.7^{\circ} \pm$ $7.4^{\circ}$ and the mean AP-T1MTA improved from $26.2^{\circ} \pm 7.8^{\circ}$ to $12.6^{\circ} \pm 7.2^{\circ}$, showing statistically significant improvement (Table 1). L-T1MTA and L-CPA (parameters for medial longitudinal arch) and AP-TCA and L-TCA (parameters for heel valgus) also improved significantly $(p<0.05)$.

\section{Physical Examination and Linear Parameters}

The mean ankle plantar flexion APF and ADKF increased without showing statistical significance $(p>0.05)$ (Table 2). However, ADKE significantly increased from $0.8^{\circ} \pm 8.5^{\circ}$ preoperatively to $13.3^{\circ} \pm 7.6^{\circ}$ postoperatively $(p<0.05)$. There was no significant difference in walking velocity, stride, or cadence before and after surgery $(p>0.05)$.

\section{Kinematics of the Pelvis, Hip, and Knee}

There were no significant changes in most of the kinematics of the pelvis and hip joint after surgery (Fig. 3). The

\section{Table 1. Radiographic Parameters in Included Patients}

\begin{tabular}{|c|c|c|c|}
\hline Parameter & Preoperative $\left({ }^{\circ}\right)$ & Postoperative $\left({ }^{\circ}\right)$ & $p$-value \\
\hline \multicolumn{4}{|l|}{ Forefoot abduction } \\
\hline AP-TNA & $32.7 \pm 11.79$ & $16.7 \pm 7.39$ & $<0.01$ \\
\hline AP-T1MTA & $26.2 \pm 7.75$ & $12.6 \pm 7.24$ & $<0.01$ \\
\hline \multicolumn{4}{|c|}{ Medial longitudinal arch } \\
\hline L-T1MTA & $34.5 \pm 8.99$ & $14.4 \pm 8.00$ & $<0.01$ \\
\hline L-CPA & $9.8 \pm 5.22$ & $19.7 \pm 4.92$ & $<0.01$ \\
\hline \multicolumn{4}{|l|}{ Heel valgus } \\
\hline AP-TCA & $34.4 \pm 11.72$ & $27.5 \pm 5.10$ & $<0.01$ \\
\hline L-TCA & $58.3 \pm 7.53$ & $48.3 \pm 4.60$ & $<0.01$ \\
\hline
\end{tabular}

Values are presented as mean \pm standard deviation.

AP-TNA: anteroposterior talonavicular angle, AP-T1MTA: anteroposterior talo-first metatarsal angle, L-T1MTA: lateral T1MTA (Meary angle), L-CPA lateral calcaneal pitch angle, AP-TCA: anteroposterior talocalcaneal angle, L-TCA: lateral talocalcaneal angle. maximum hip extension in terminal stance in sagittal plane was $6.27^{\circ} \pm 8.6^{\circ}$ preoperatively and $0.68^{\circ} \pm 8.8^{\circ}$ postoperatively $(p<0.05)$. There was no statistically significant difference between preoperative and postoperative mean valgus, flexion, and external rotation of the knee joint. Among the kinematic results of the knee joint, only the peak knee extension in stance significantly improved from $-0.61^{\circ} \pm 4.17^{\circ}$ preoperatively to $4.04^{\circ} \pm 7.85^{\circ}$ postoperatively $(p<0.05)$.

\begin{tabular}{|c|c|c|}
\hline Ankle kinematics & $\begin{array}{c}\text { Mean } \pm \text { standard } \\
\text { deviation }\left({ }^{\circ}\right)\end{array}$ & $p$-value* \\
\hline Varus (+) - valgus (-) & & $<0.01$ \\
\hline Preoperative & $-35.48 \pm 14.48$ & \\
\hline Postoperative & $-16.64 \pm 13.58$ & \\
\hline Dorsiflexion (+) - plantar flexion (-) & & 0.08 \\
\hline Preoperative & $-1.18 \pm 12.08$ & \\
\hline Postoperative & $3.85 \pm 5.50$ & \\
\hline
\end{tabular}

Internal rotation (+) - external rotation (-)

0.47

$\begin{array}{ll}\text { Preoperative } & -5.22 \pm 13.63 \\ \text { Postoperative } & -2.61 \pm 10.46\end{array}$

Peak dorsiflexion in midstance phase

Preoperative

$9.25 \pm 13.11$

Postoperative

$15.16 \pm 7.19$

Peak dorsiflexion in swing phase

0.29

$\begin{array}{ll}\text { Preoperative } & 0.97 \pm 11.26 \\ \text { Postoperative } & 3.67 \pm 3.91\end{array}$

Foot progression angle

0.04

Preoperative

$-20.31 \pm 7.89$

Postoperative

$-14.21 \pm 12.09$

Peak plantar flexion in terminal stance phase

$<0.01$

$\begin{array}{lc}\text { Preoperative } & -13.50 \pm 12.18 \\ \text { Postoperative } & -3.71 \pm 4.50\end{array}$

Range of ankle joint motion in sagittal plane

$<0.01$

$\begin{array}{ll}\text { Preoperative } & 22.75 \pm 5.90 \\ \text { Postoperative } & 11.29 \pm 10.64\end{array}$

${ }^{*} p<0.05$, statistically significant. 
Kim et al. Effect of Calcaneal Lengthening in Idiopathic Flexible Flatfoot

Clinics in Orthopedic Surgery • Vol. 12, No. 3, $2020 \bullet$ www.ecios.org
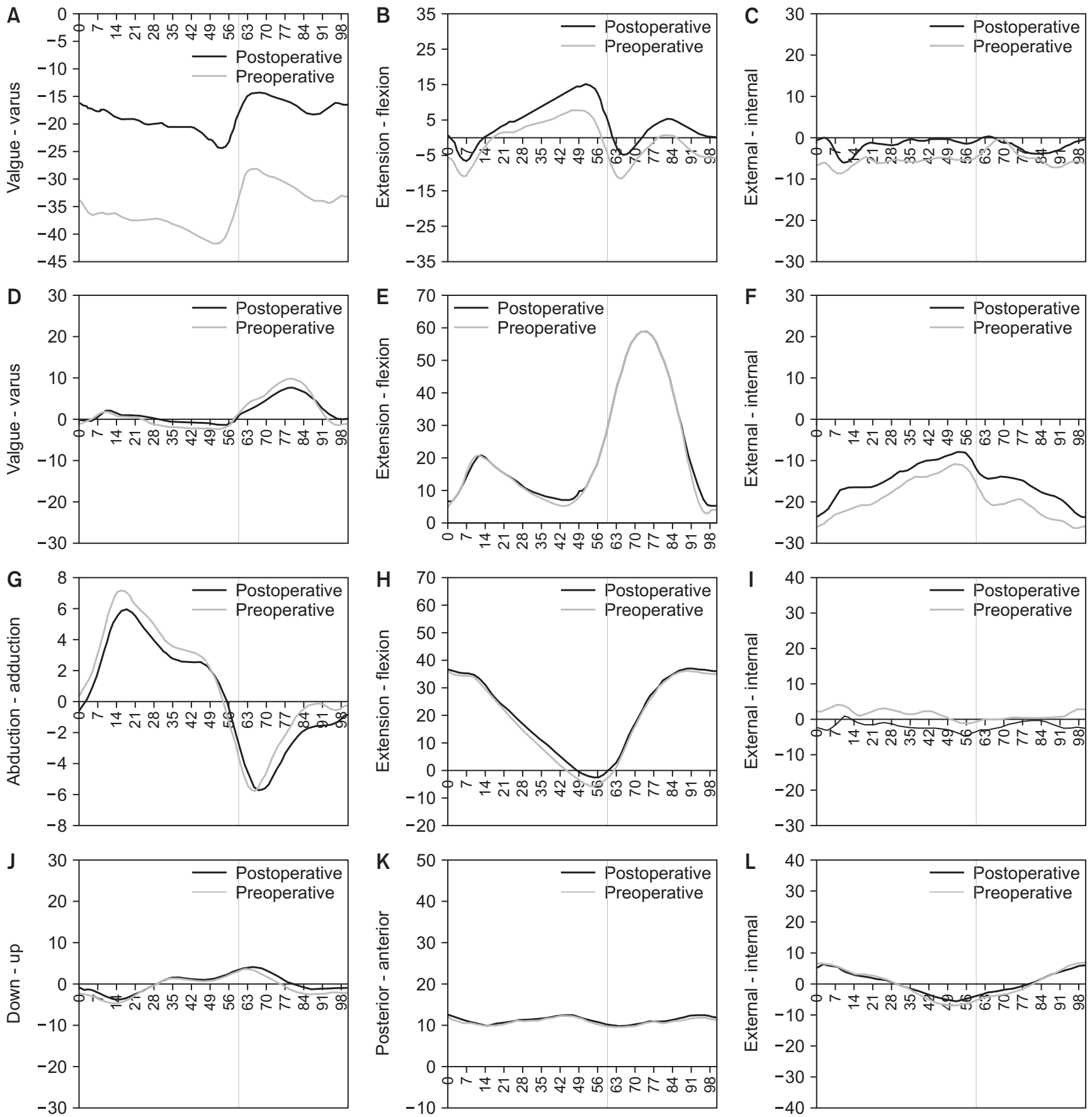

Fig. 3. Preoperative and postoperative kinematics of the lower extremity. (A) Coronal plane ankle. (B) Sagittal plane ankle. (C) Transverse plane ankle. (D) Coronal plane knee. (E) Sagittal plane knee. (F) Transverse plane knee. (G) Coronal plane hip. (H) Sagittal plane hip. (I) Transverse plane hip. (J) Coronal plane pelvis. (K) Sagittal plane pelvis. (L) Transverse plane pelvis.

\section{Kinematics of the Ankle Joint}

The mean values of ankle kinematics in the sagittal plane and transverse plane were not significantly different between preoperative and postoperative assessments $(p<$ 0.05 ). The mean ankle valgus on the coronal plane, however, was $35.5^{\circ} \pm 14.5^{\circ}$ preoperatively and $16.6^{\circ} \pm 13.6^{\circ}$ postoperatively $(p<0.05)$ (Table 2 , Fig. 3 ). The maximal ankle dorsiflexion at the end of the second rocker increased from $9.3^{\circ} \pm 13.1^{\circ}$ preoperatively to $15.2^{\circ} \pm 7.2^{\circ}$ postoperatively $(p>0.05)$. The maximum plantar flexion at the end of the third rocker was $13.5^{\circ} \pm 12.2^{\circ}$ of plantar flexion preoperatively and $3.7^{\circ} \pm 4.5^{\circ}$ postoperatively $(p<0.05)$. 
Kim et al. Effect of Calcaneal Lengthening in Idiopathic Flexible Flatfoot

Clinics in Orthopedic Surgery • Vol. 12, No. 3, $2020 \bullet$ www.ecios.org

The range of motion of the ankle (maximum plantar flexion and maximal dorsiflexion) during walking was $22.8^{\circ} \pm$ $5.9^{\circ}$ preoperatively and $11.3^{\circ} \pm 10.6^{\circ}$ postoperatively $(p<$ $0.05)$. As forefoot abduction was corrected, foot progression angle was improved from $20.3^{\circ} \pm 7.9^{\circ}$ to $14.2^{\circ} \pm 12.1^{\circ}$ of external rotation $(p<0.05)$.

\section{Kinetics}

The kinetic results obtained by the gait analysis were divided by the body weight of the patient and converted to moment per $\mathrm{kg}(\mathrm{Nm} / \mathrm{kg})$ and power per $\mathrm{kg}(\mathrm{J} / \mathrm{sec} / \mathrm{kg}=\mathrm{W} /$ $\mathrm{kg}$ ) for statistical analysis. The maximum plantar flexion moment on the sagittal plane increased from $0.66 \pm 0.24$ preoperatively to $0.83 \pm 0.24$ postoperatively $(p<0.05)$
(Table 3, Fig. 4). The maximum moment on the transverser plane, which reflects the inefficient moment due to the forefoot valgus, decreased from $0.13 \pm 0.07$ preoperatively to $0.06 \pm 0.05$ postoperaitvley $(p<0.05)$. The maximum ankle power increased from $1.28 \pm 0.60$ preoperatively to $1.44 \pm 0.38$ postoperatively, but the change was not statistically significant $(p>0.05)$. However, the minimum power and power range of the ankle joint showed a statistically significant difference $(p<0.05)$, and the overall range of the ankle showed a marked increase on the graph.

On the coronal plane, the maximal valgus moment of the knee joint was $0.24 \pm 0.06 \mathrm{Nm} / \mathrm{kg}$ preoperatively and $0.32 \pm 0.09 \mathrm{Nm} / \mathrm{kg}$ postoperatively $(p<0.05)$ (Table $3)$. The minimum extension moment of knee joint on the

Table 3. Kinetic Results of the Ankle and Knee Joints

\begin{tabular}{|c|c|c|c|c|c|}
\hline \multirow{2}{*}{\multicolumn{2}{|c|}{ Parameter }} & \multicolumn{2}{|c|}{ Ankle joint } & \multicolumn{2}{|c|}{ Knee joint } \\
\hline & & \multirow{2}{*}{ Moment (Nm/kg) } & \multirow[t]{2}{*}{ Power (W/kg) } & \multirow{2}{*}{ Moment (Nm/kg) } & \multirow{2}{*}{ Power (W/kg) } \\
\hline \multicolumn{2}{|l|}{ Sagittal plane } & & & & \\
\hline \multirow[t]{2}{*}{$\operatorname{Max} \pm$ SD } & Preoperative & $0.66 \pm 0.24$ & $1.28 \pm 0.60$ & $0.55 \pm 0.23$ & $0.73 \pm 0.23$ \\
\hline & Postoperative & $0.83 \pm 0.24^{*}$ & $1.44 \pm 0.38$ & $0.57 \pm 0.23$ & $0.76 \pm 0.13$ \\
\hline \multirow[t]{2}{*}{$\operatorname{Min} \pm S D$} & Preoperative & $-0.19 \pm 0.12$ & $-0.70 \pm 0.40$ & $-0.28 \pm 0.07$ & $-1.07 \pm 0.57$ \\
\hline & Postoperative & $-0.20 \pm 0.16$ & $-1.01 \pm 0.47^{*}$ & $-0.22 \pm 0.04^{*}$ & $-1.10 \pm 0.40$ \\
\hline \multirow[t]{2}{*}{ Range $\pm S D$} & Preoperative & $0.85 \pm 0.33$ & $1.92 \pm 0.69$ & $0.82 \pm 0.24$ & NA \\
\hline & Postoperative & $1.03 \pm 0.33$ & $2.45 \pm 0.21^{*}$ & $0.79 \pm 0.21$ & NA \\
\hline \multicolumn{6}{|l|}{ Coronal plane } \\
\hline \multirow[t]{2}{*}{$\operatorname{Max} \pm$ SD } & Preoperative & $0.08 \pm 0.02$ & NA & $0.24 \pm 0.06$ & NA \\
\hline & Postoperative & $0.07 \pm 0.07$ & NA & $0.32 \pm 0.09^{*}$ & NA \\
\hline \multirow[t]{2}{*}{$\operatorname{Min} \pm S D$} & Preoperative & $0.09 \pm 0.09$ & NA & $-0.05 \pm 0.02$ & NA \\
\hline & Postoperative & $0.09 \pm 0.08$ & NA & $-0.06 \pm 0.02$ & NA \\
\hline \multirow[t]{2}{*}{ Range \pm SD } & Preoperative & $0.16 \pm 0.07$ & NA & $0.30 \pm 0.07$ & NA \\
\hline & Postoperative & $0.16 \pm 0.08$ & NA & $0.38 \pm 0.09^{*}$ & NA \\
\hline \multicolumn{6}{|c|}{ Transverse plane } \\
\hline \multirow[t]{2}{*}{$\operatorname{Max} \pm S D$} & Preoperative & $0.13 \pm 0.07$ & NA & $0.05 \pm 0.02$ & NA \\
\hline & Postoperative & $0.06 \pm 0.05^{*}$ & NA & $0.06 \pm 0.03$ & NA \\
\hline \multirow[t]{2}{*}{$\operatorname{Min} \pm S D$} & Preoperative & $-0.64 \pm 0.16$ & NA & $-0.24 \pm 0.06$ & NA \\
\hline & Postoperative & $-0.20 \pm 0.16^{*}$ & NA & $-0.32 \pm 0.09^{*}$ & NA \\
\hline \multirow[t]{2}{*}{ Range \pm SD } & Preoperative & $0.78 \pm 0.14$ & NA & $0.30 \pm 0.07$ & NA \\
\hline & Postoperative & $0.26 \pm 0.17^{*}$ & NA & $0.38 \pm 0.09^{*}$ & NA \\
\hline
\end{tabular}

$\mathrm{W}: 1 \mathrm{~J} / \mathrm{sec}$, max: maximum value, min: minimum value, SD: standard deviation, NA: not available. ${ }^{*} p<0.05$. 
Kim et al. Effect of Calcaneal Lengthening in Idiopathic Flexible Flatfoot

Clinics in Orthopedic Surgery • Vol. 12, No. 3, $2020 \bullet$ www.ecios.org
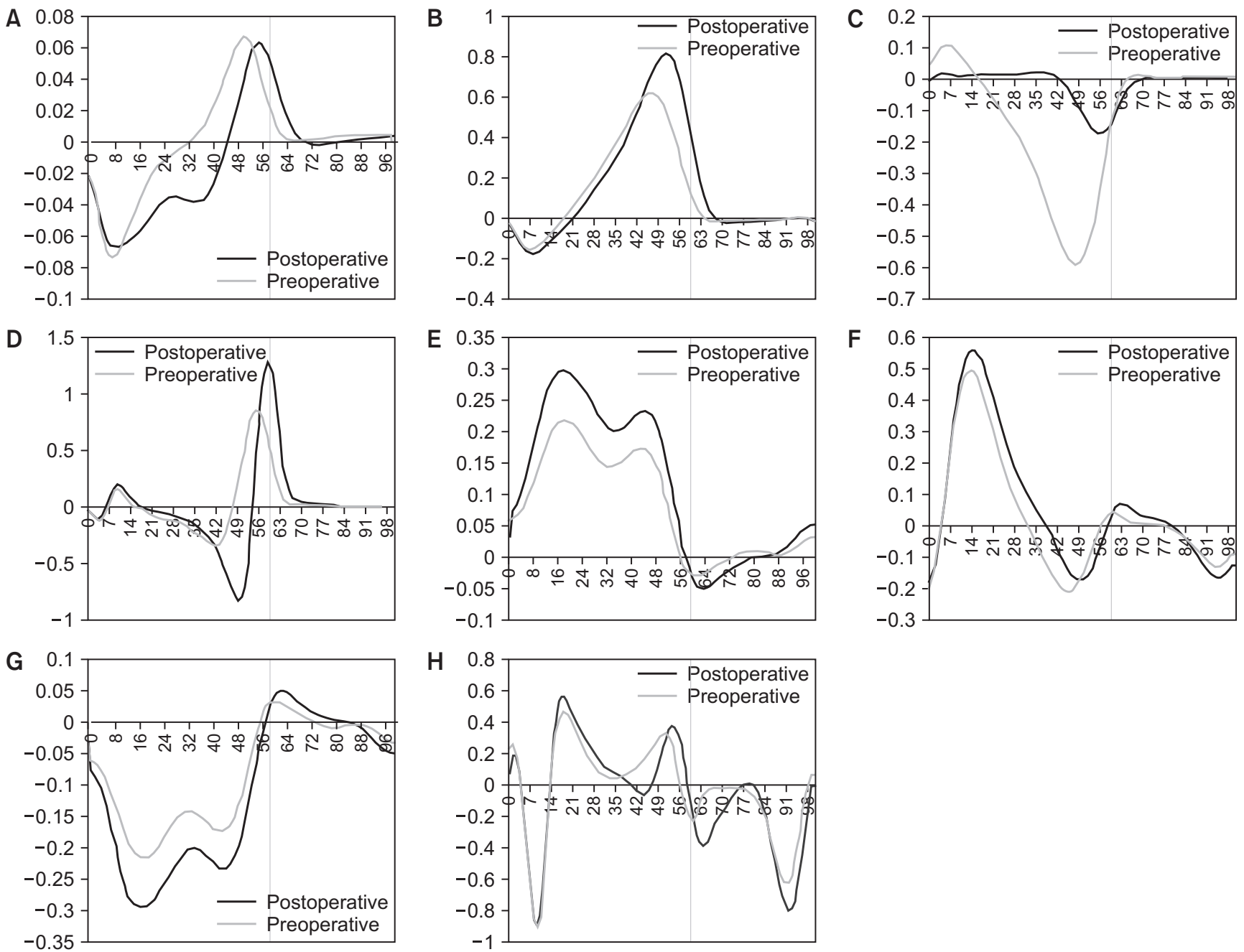

Fig. 4. Preoperative and postoperative kinetics of the lower extremity. (A) Coronal plane ankle moment. (B) Sagittal plane ankle moment. (C) Transverse plane ankle moment. (D) Sagittal plane knee moment. (E) Coronal plane knee moment. (F) Sagittal plane knee moment. (G) Transverse plane knee moment. (H) Sagittal plane knee moment.

sagittal plane during the swing phase increased from -0.28 \pm 0.07 preoperatively to $-0.22 \pm 0.04$ postoperatively $(p<$ $0.05)$.

\section{Clinical Examinations}

The mean Yoo score was $9.2 \pm 1.05$ postoperatively: satisfactory in 24 cases (86\%) and unsatisfactory in 4 cases. Of the unsatisfactory 4 cases, 2 cases were overcorrection and 2 cases were undercorrection, and 3 of them were well managed by wedge foot insoles. But 1 case with severe joint laxity (Wynne-Davies criteria 4) suffered callosity and pain, and she underwent triple arthrodesis. ${ }^{13)}$

The most common complication was postoperative pain. Thirteen cases complained of pain, which was managed by medication and resolved within 6 months in 10 cases. Three cases complained of mild pain at postoperative
1 year. Pain that lasted for 1 year after the operation was due to the overcorrection and irritation by the implant used for fixation. Postoperatively, 11 cases (39\%) showed subluxation of calcaneus-cuboid joint at an angle of $31^{\circ} \pm 6^{\circ}$, which substantially decreased at the 1-year follow-up. Fortunately, there was no postoperative infection and nonunion.

\section{DISCUSSION}

A number of studies have reported the results of CLO for flexible flatfoot caused by neuromuscular diseases such as cerebral palsy and Charcot-Marie-Tooth disease..$^{9-12,14,15)}$ They showed that 3-dimensional radiologic parameters were effectively improved after CLO. The current study was conducted on patients with symptomatic flexible flatfoot without neuromuscular diseases. The radiologic 
Kim et al. Effect of Calcaneal Lengthening in Idiopathic Flexible Flatfoot

Clinics in Orthopedic Surgery • Vol. 12, No. 3, $2020 \bullet$ www.ecios.org

results of this study showed that 3-dimensional deformity of flexible flatfoot was corrected after CLO. Radiologic parameters for forefoot abduction (AP-TNA and AP-T1MTA), medial longitudinal arch (L-T1MTA and L-CPA), and heel valgus (AP-TCA and L-TCA) were all corrected significantly after CLO $(p<0.05)$.

Yoo et al. ${ }^{10)}$ and Sung et al. ${ }^{16)}$ pointed out that an additional medial surgery is needed depending on the degree of preoperative deformity. In addition, Park et al. ${ }^{17)}$ also reported that CLO and peroneus brevis lengthening can effectively correct pes planovalgus deformity in children with cerebral palsy, and that additional surgery on the medial side might be needed to reduce undercorrection for severe deformities such as AP-T1MTA $>23^{\circ}$, L-T1MTA $>36^{\circ}$, and naviculocuboid overlap $>72 \%$. On the other hand, Moraleda et al. ${ }^{18)}$ reported that additional procedures other than CLO had the effect of improving several radiological parameters but not the clinical outcome.

In this study, CLO was done for the patients whose deformity was passively correctable, but undercorrection or overcorrection of the deformity was observed in 4 cases. Severe deformity, ligamentous laxity, concomitant deformity (in particular, forefoot pronation or supination), and inappropriate surgical procedure may cause inadequate correction. In this study, surgically corrected calcaneus was internally fixed to prevent undercorrection. Preoperatively, fixed forefoot supination should be checked carefully, as this hidden deformity can cause varus deformity postoperatively, which is an important factor of overcorrection.

Yoo et al. ${ }^{10)}$ reported the results of gait analysis after CLO in cerebral palsy children and showed the decrease of the foot progression angle and maximum dorsiflexion angle during stance phase and the increase of ankle power at push-off. Kadhim and Miller ${ }^{19)}$ also reported the results of CLO: there were changes in foot progression angle (18.8 to $10.2^{\circ}$ ), ankle max dorsiflexion $\left(13.9^{\circ}\right.$ to $\left.16.1^{\circ}\right)$, and maximum knee extension at stance $\left(-12.3^{\circ}\right.$ to $\left.-15.6^{\circ}\right)$ in cerebral palsy children with crouch gait. Both studies reported conflicting results for maximum dorsiflexion angle.

Yoo et al. ${ }^{10)}$ and Kadhim and Miller ${ }^{19)}$ reported conflicting results on the degree of maximum dorsiflexion during stance phase. In our study, although the amount of dorsiflexion was increased, it was not statistically significant. And the sagittal plane graph of the ankle joint was shifted to the dorsiflexion side, and the amount of maximum dorsiflexion was increased. These results reflect the presence of selection bias: patients with heel cord lengthening were included as in the study by Kadhim and Miller. ${ }^{19)}$ In other words, due to heel cord lengthening, the overall degree of ankle dorsiflexion was considered to have increased in the postoperative gait analysis.

The ankle joint showed many kinematic changes after surgery. In particular, the degree of heel valgus decreased significantly in the coronal plane. On the transverse plane, the foot progression angle improved from outtoeing $20.31^{\circ}$ to out-toeing $14.21^{\circ}$ as a result of correction of the forefoot abduction, which are similar to the results reported by Yoo et al. ${ }^{10)}$ and Kadhim and Miller. ${ }^{19)}$ These kinematic changes showed that the static foot deformity was corrected morphologically after CLO as observed in radiographs, but also the corrected foot shape was well maintained even during gait.

The decrease in minimum hip flexion and maximum knee extension in the stance phase is interpreted to be due to the proper dorsiflexion of the ankle joint by Achilles tendon lengthening rather than by the calcaneal lengthening. Achilles tendon lengthening seems to have resulted in proper dorsiflexion of the ankle joint and decrease in inadequate compensatory movement in the proximal joint. In the study of Stebbins et al., spontaneous correction of hip joint motion occurred during the gait after foot surgery in cerebral palsy patients. ${ }^{20)}$ It showed the possibility of positive change in proximal kinematics by correction of distal deformity. In the current study, varus moment was expected to decrease as the hindfoot valgus was corrected on the coronal plane, showing a marked decrease in the graph, but it was not statistically significant. If statistical processing is performed by dividing the stance interval, it might be expected that there will be a significant difference at the midstance and terminal stance.

Kim et al. ${ }^{7)}$ and Yoo et al. ${ }^{10)}$ reported on the kinetics of flatfoot. Kim et al. ${ }^{7)}$ reported a statistically significant decrease in the moment $(0.88 \pm 0.37)$ and power $(1.40 \pm$ 0.79 ) on the sagittal plane of the third rocker with pushoff in the flexible flatfoot. Yoo et al. ${ }^{10)}$ reported increased power during push-off after surgery. In our study, the maximum plantar flexion moment at push-off on the sagittal plane increased from $0.66 \mathrm{Nm} / \mathrm{kg}$ before surgery to $0.83 \mathrm{Nm} / \mathrm{g}$ after surgery $(p<0.05)$. The power on sagittal plane increased both maximum energy production (from $1.28 \mathrm{~W} / \mathrm{kg}$ to $1.44 \mathrm{~W} / \mathrm{kg} ; p>0.05$ ) and maximum energy absorption (from $-0.70 \mathrm{~W} / \mathrm{kg}$ to $-1.01 \mathrm{~W} / \mathrm{kg} ; p<0.01$ ) on the graph. As the medial longitudinal arch was corrected and the lever arm of the foot became solid, the push-off moment and power on sagittal plane increased. On the other hand, the maximum moment, the minimum moment, and the range of power decreased on the transverse and coronal planes. It can be interpreted that the unnecessary moments were reduced other than on the sagittal plane as the forefoot abduction and heel valgus decreased. 
Kim et al. Effect of Calcaneal Lengthening in Idiopathic Flexible Flatfoot

Clinics in Orthopedic Surgery • Vol. 12, No. 3, $2020 \bullet$ www.ecios.org

As a result, the foot progression angle and hindfoot valgus were corrected by CLO, which reduced the unnecessary varus moment and rotational moment. This study is different from the previous studies in that the cause of flexible flatfoot of the subjects in this study was idiopathic rather than the neuromuscular cause.

This study has some limitations. First, since various procedures were used for each patient, the patient's postoperative changes may not have resulted from CLO alone. Second, the postoperative gait analysis of this study was performed at approximately 1 year after surgery when the patient was admitted to the hospital to remove the internal fixation. Metals often protruded beneath the skin, leading to irritation symptoms. Therefore, there is a possibility that the remaining metals at the time of gait analysis might have influenced the gait results. Third, there is a limitation inherent to the gait analysis for assessing foot deformity, as the foot is regarded as a solid body for gait analysis.
In conclusion, 3-dimensional deformities of the flexible flatfoot were effectively corrected after CLO, based on morphologic assessment in radiographic evaluation. The correction was well maintained during gait based on the kinematics results at 1 year after surgery. Heel valgus was reduced, and forefoot abduction and external foot progression angle were internally rotated during gait after CLO. Improvements in lever arm dysfunction after the deformity correction reduced the consuming moments on both the coronal and transverse planes and increased the push-off moment on the sagittal plane, which improved the overall energy efficiency for walking.

\section{CONFLICT OF INTEREST}

No potential conflict of interest relevant to this article was reported.

\section{REFERENCES}

1. Harris RI, Beath T. Hypermobile flat-foot with short tendo achillis. J Bone Joint Surg Am. 1948;30(1):116-40.

2. Andreacchio A, Orellana CA, Miller F, Bowen TR. Lateral column lengthening as treatment for planovalgus foot deformity in ambulatory children with spastic cerebral palsy. J Pediatr Orthop. 2000;20(4):501-5.

3. Carson MC, Harrington ME, Thompson N, O'Connor JJ, Theologis TN. Kinematic analysis of a multi-segment foot model for research and clinical applications: a repeatability analysis. J Biomech. 2001;34(10):1299-307.

4. Dogan A, Albayrak M, Akman YE, Zorer G. The results of calcaneal lengthening osteotomy for the treatment of flexible pes planovalgus and evaluation of alignment of the foot. Acta Orthop Traumatol Turc. 2006;40(5):356-66.

5. Bertani A, Cappello A, Benedetti MG, Simoncini L, Catani F. Flat foot functional evaluation using pattern recognition of ground reaction data. Clin Biomech (Bristol, Avon). 1999; 14(7):484-93.

6. Mosca VS. Calcaneal lengthening for valgus deformity of the hindfoot. Results in children who had severe, symptomatic flatfoot and skewfoot. J Bone Joint Surg Am. 1995;77(4):500-12.

7. Kim HY, Shin HS, Ko JH, Cha YH, Ahn JH, Hwang JY. Gait analysis of symptomatic flatfoot in children: an observational study. Clin Orthop Surg. 2017;9(3):363-73.

8. Crego CH Jr, Ford LT. An end-result of various operative procedures for correcting flat feet in children. J Bone Joint Surg Am. 1952;34(1):183-95.
9. Sung KH, Kwon SS, Chung CY, Lee KM, Park MS. Radiographic changes of the mid-tarsal joint after calcaneal lengthening for planovalgus foot deformity. Foot Ankle Surg. 2020;26(1):110-5.

10. Yoo WJ, Chung CY, Choi IH, Cho TJ, Kim DH. Calcaneal lengthening for the planovalgus foot deformity in children with cerebral palsy. J Pediatr Orthop. 2005;25(6):781-5.

11. Noritake K, Yoshihashi Y, Miyata T. Calcaneal lengthening for planovalgus foot deformity in children with spastic cerebral palsy. J Pediatr Orthop B. 2005;14(4):274-9.

12. Zeifang F, Breusch SJ, Doderlein L. Evans calcaneal lengthening procedure for spastic flexible flatfoot in 32 patients (46 feet) with a followup of 3 to 9 years. Foot Ankle Int. 2006; 27(7):500-7.

13. Wynne-Davies R. Familial joint laxity. Proc R Soc Med. 1971;64(6):689-90.

14. Danko AM, Allen B Jr, Pugh L, Stasikelis P. Early graft failure in lateral column lengthening. J Pediatr Orthop. 2004; 24(6):716-20.

15. Adams SB Jr, Simpson AW, Pugh LI, Stasikelis PJ. Calcaneocuboid joint subluxation after calcaneal lengthening for planovalgus foot deformity in children with cerebral palsy. J Pediatr Orthop. 2009;29(2):170-4.

16. Sung KH, Chung CY, Lee KM, Lee SY, Park MS. Calcaneal lengthening for planovalgus foot deformity in patients with cerebral palsy. Clin Orthop Relat Res. 2013;471(5):1682-90.

17. Park KB, Park HW, Lee KS, Joo SY, Kim HW. Changes in 
Kim et al. Effect of Calcaneal Lengthening in Idiopathic Flexible Flatfoot

Clinics in Orthopedic Surgery • Vol. 12, No. 3, $2020 \bullet$ www.ecios.org

dynamic foot pressure after surgical treatment of valgus deformity of the hindfoot in cerebral palsy. J Bone Joint Surg Am. 2008;90(8):1712-21.

18. Moraleda L, Salcedo M, Bastrom TP, Wenger DR, Albinana J, Mubarak SJ. Comparison of the calcaneo-cuboid-cuneiform osteotomies and the calcaneal lengthening osteotomy in the surgical treatment of symptomatic flexible flatfoot. J Pediatr Orthop. 2012;32(8):821-9.
19. Kadhim M, Miller F. Crouch gait changes after planovalgus foot deformity correction in ambulatory children with cerebral palsy. Gait Posture. 2014;39(2):793-8.

20. Stebbins J, Harrington M, Thompson N, Zavatsky A, Theologis T. Gait compensations caused by foot deformity in cerebral palsy. Gait Posture. 2010;32(2):226-30. 